\title{
Study of the evolution of the mechanical properties of orthodontic arches by stimulated infrared thermography
}

\author{
by N. Chahine*, K.Mouhoubi**, A. Diakhate ${ }^{* *}$, S. Harakeh ${ }^{* * *}$, P. Millet* ${ }^{*}$ JL. Bodnar ${ }^{* *}$ \\ * Univ. Reims Champagne Ardenne, LISM EA4695, UFR Odontologie - 51100 Reims - CHU Reims, Pôle de \\ Médecine Bucco-Dentaire, F-51100 Reims, France. \\ ${ }^{* *}$ GRESPI / CATHERM, UFR Sciences Exactes et Naturelles, BP 1039, 51687 Reims cedex 02, France \\ *** U.F.R. ODONTOLOGIE, 2, rue du Général Koenig, 51100 REIMS, France \\ **** Steve Harakeh, Special Infectious Agents Unit, King Fahd Medical Research Center, King Abdulaziz \\ University, Jeddah, Kingdom of Saudi Arabia
}

\begin{abstract}
Electron microscopic observation of the surface of orthodontic arches reveals observable differences between new and used arches after four weeks in the mouth. This qualitative observation led us to consider their study by stimulated infrared thermography. These dental arches consist of a nickel-titanium (NiTi) shape memory alloy and are highly stressed during the stay in the mouth. Over the months, there will be evolution of their mechanical properties with multifactorial causes. In this study, we will demonstrate that it is possible to differentiate between the used arcs from the new arcs by observing their thermal response during a controlled heating.
\end{abstract}

\section{Introduction}

This study focuses on the analysis of alterations affecting orthodontic arches. These orthodontic arches are used in dentistry to correct the positioning of the teeth. These devices must have remarkable strength and elastic properties. They are generally made of a nickel and titanium alloy called NiTi. This shape memory alloy allows to exert a constant pressure on the tooth and thus corrects its position during treatment. The duration of this orthodontic treatment is of the order of 12 to 15 months, it is more expensive because of the frequent replacement of the arches.

The oral medium exposes the arc to many stresses and constraints, including both mechanical and chemical (acidity, tartar ...). Such conditions will alter its properties and require its regular replacement. In general, the bow remains in the mouth for 4 weeks before being replaced. The renewal of the arches makes the treatment expensive due to the number of interventions needed. Based on this, it would be interesting to identify the causes of such alterations occurring in these devices and deal with them.

In this work, we will identify the defects using a control method called infrared thermography. The advantages of this method are numerous: one of the interests lies in its ease of implementation and in its limited cost. Moreover, this method is non-destructive and allows the repetition of the same tests. Finally, it allows the analysis of defects on the surface when the analysis is carried out in both a static mode and more in depth if this analysis is carried out in a dynamic mode. This is why the analysis of the results will be conducted in two stages: a first stage include analysis of static (without heat input) and a second stage in dynamic mode (with heat input). The chosen approach will allow the comparison between the thermal responses of new and used samples when subjected to a thermal excitation or not. This protocol will make it possible to identify the altered characteristics in the samples and will make it possible to demonstrate the correlation between the presence of defects and the specific characteristics of these samples. The final goal is to find solutions for those alterations.

\section{Device and experimental protocol}

\subsection{Device}

\subsubsection{Static analysis}

The sample was attached at both ends using two connectors (see Figure 1). The observation is carried out using a Flir thermal imaging camera (model SC655) clocked at $6.25 \mathrm{~Hz}$ when the sample is in thermal equilibrium with the ambient environment. 


\subsubsection{Dynamic analysis}

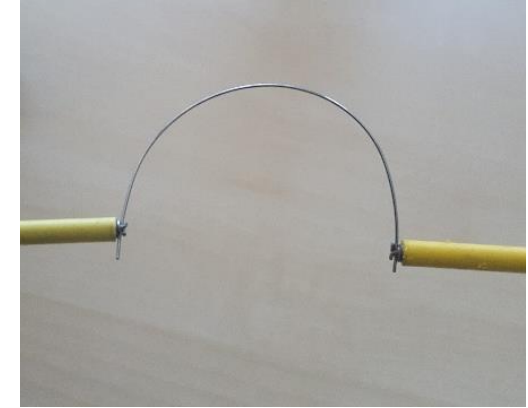

Fig. 1. Arc fixed on the device

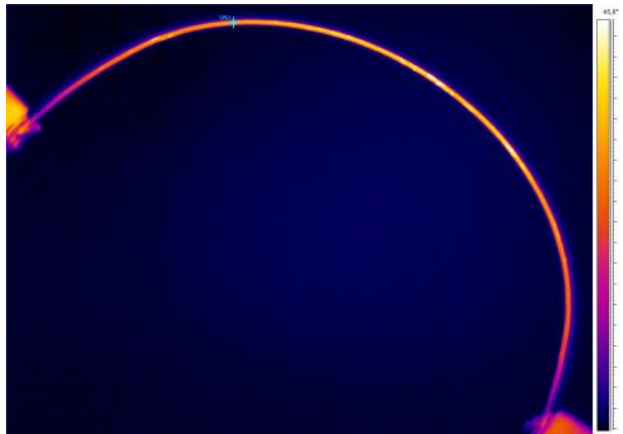

Fig. 3. Thermal image of an electric current arc

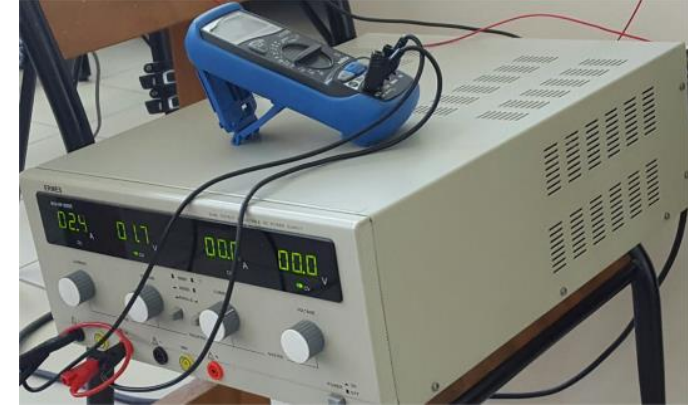

Fig. 2. Stabilized power supply

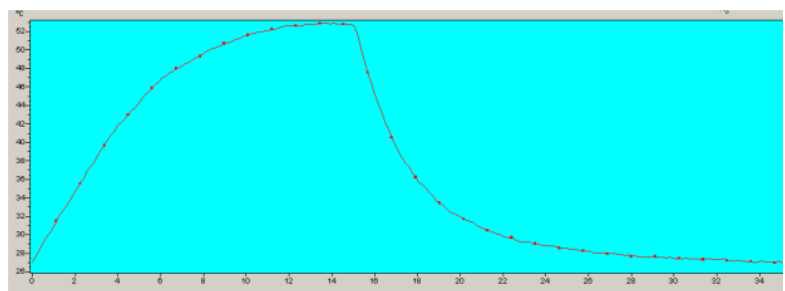

Fig. 4. Temperature response of an arc in dynamic regime

The arc is maintained with the same connectors but is traversed by an electric current delivered by a stabilized power supply (see Figures 1 and 2). The dental arch serves as an electrical resistance, the observation of its heating by joule effect with a thermal camera allows studying it in dynamic regime. The thermally stressed section (located between the 2 terminals) has a length of $50 \mathrm{~mm}$ for all the samples studied. A voltage of $1.7 \mathrm{~V}$ at $2.4 \mathrm{~A}$ (ie a power of $4 \mathrm{~W}$ ) was applied to the portion of the arc for a period of 15 seconds. The total observation time is 35 seconds, 15 seconds of heating and 20 seconds for cooling of the sample. The thermal transition makes it possible to highlight the defects present in the material.

The observation is carried out as before using a thermal camera (FLIR, equipped with a 2.8-fold macro lens), driven by thermal imaging software (ThermaCAM Researcher Pro 2.01), clocked at an acquisition frequency of $6.25 \mathrm{~Hz}$.

\subsection{Experimental protocol}

The objective was to identify and quantify the extent of alterations of orthodontic arches. These alterations may be surface defects due to oxidation or deposition zones (tartar, etc.) or internal defects such as micro-cracks or microlaminations. The causes of these defects will be discussed based on the appearance or the development of these defects. Our interest is to use infrared thermography to detect there defects and characterize them.

The analysis will be done by the extraction of the thermograms before heating (static) them or at the end of the warm-up phase (dynamic), where the temperature is the highest.

The static analysis should allow us to highlight the superficial defects characterized by emissivity variations because the samples are in thermal equilibrium with the medium and the measured signal can depend only on the emissivity parameter and the environmental conditions. While results obtained from the dynamic phase will allow us to highlight the presence of internal defects as a result of subjecting the sample to an internal heat flow related to local thermal and electrical conductivity variations induced by the presence of these defects in the sample.

The thermograms obtained using a thermal camera will allow us to carry out qualitative and quantitative analyzes. The qualitative analysis will yield a first sorting between samples but a quantitative approach is necessary to classify the samples in a rigorous and objective manner. The count of faults identified on each arc and the measurement of various parameters concerning them will be done using the open source software ImageJ and more particularly thanks to its counting module "Analysis particles" (see description in the table below) : after performing a thresholding operation on the 
thermal image, it would be possible to obtain a large number of very interesting information on the defects observed and their characteristics such as their numbers, their areas or their perimeters. This information will be useful in positioning the arcs relative to each other according to different criteria that will make them possible to cross.

Table 1. Defect counting with ImageJ

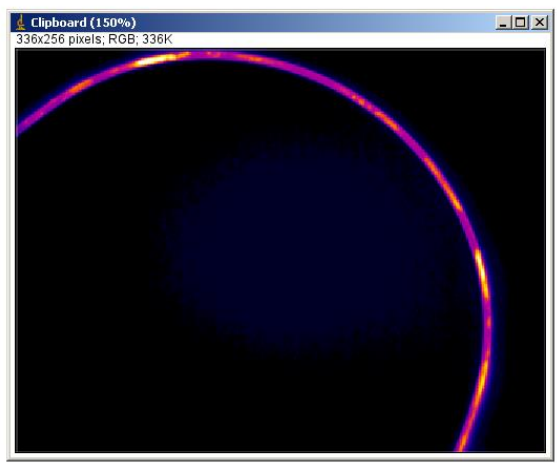

Fig. 5. Raw image

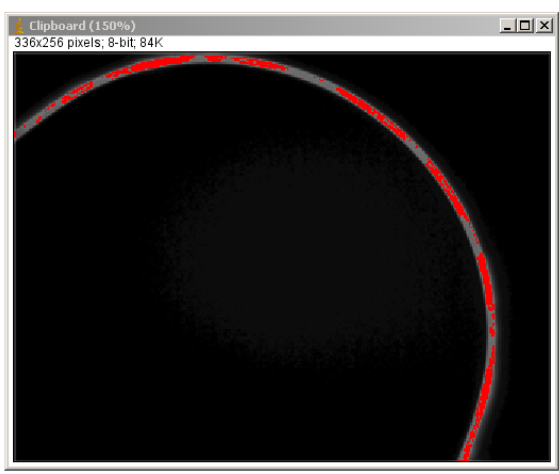

Fig. 7. thresholding

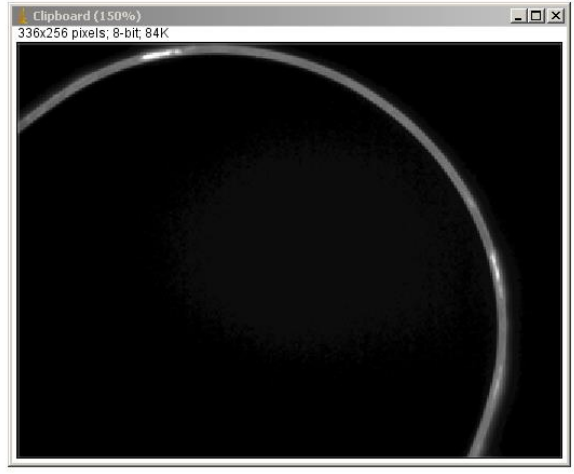

Fig. 6. 8-bit switching

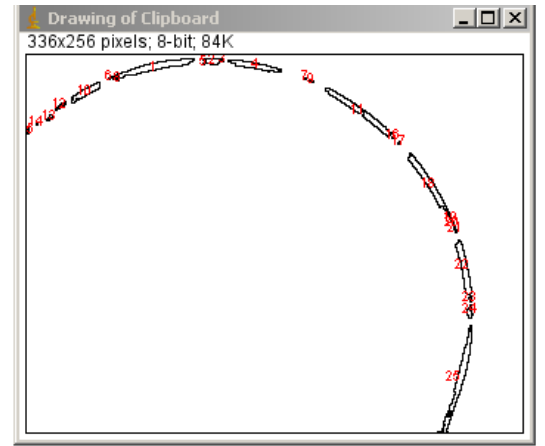

Fig. 8. Counting

\section{Presentation of the samples studied}

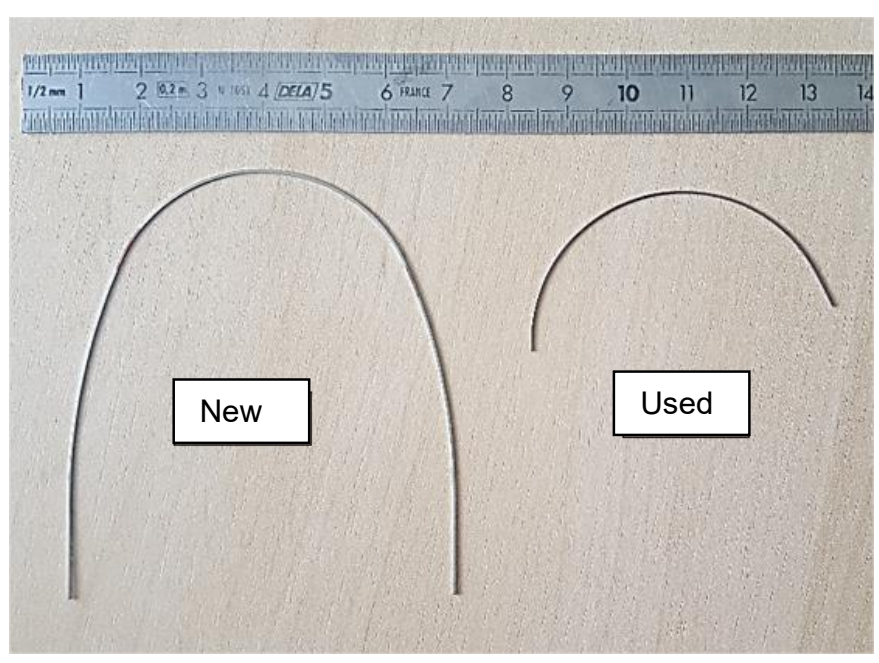

Fig. 9. New and used arches 
18 orthodontic arches were analyzed. These arches were made from nickel titanium shape memory alloy which gives them super elastic properties. There were new samples measuring $17 \mathrm{~cm}$ in length or used ones with varying lengths according to the cut made by the practitioner according to the dentition of the patient $(7$ to $17 \mathrm{~cm})$. The orthodontic arches analyzed came from different manufacturers (AZDENT, AMERICAN ORTHO, ORTHOCLASSIC), their sections varied according to two modalities: round sections of $0.16 \mathrm{~mm}$ of diameter or rectangular sections of dimensions $0.16 \times 0.22 \mathrm{~mm}$.

\section{Results obtained}

\subsection{Qualitative approach}

In this analysis we limit ourselves to a direct description of the thermograms. The goal was to observe the presence or absence of defects.

\subsubsection{Static analysis}

The 18 new and used samples were studied at room temperature (thermal equilibrium). It is shown below a comparison between new / used Orthoclassic brand arches.

Table 2. Static arc comparison

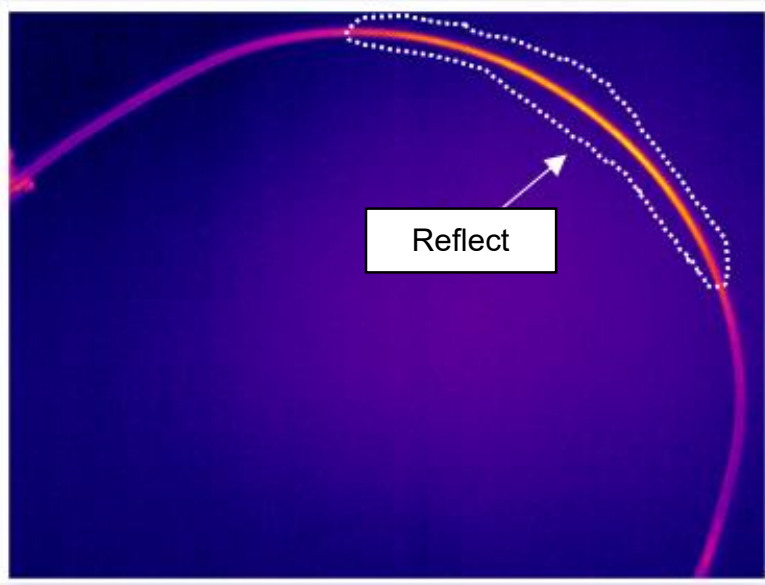

Fig. 10. New arches

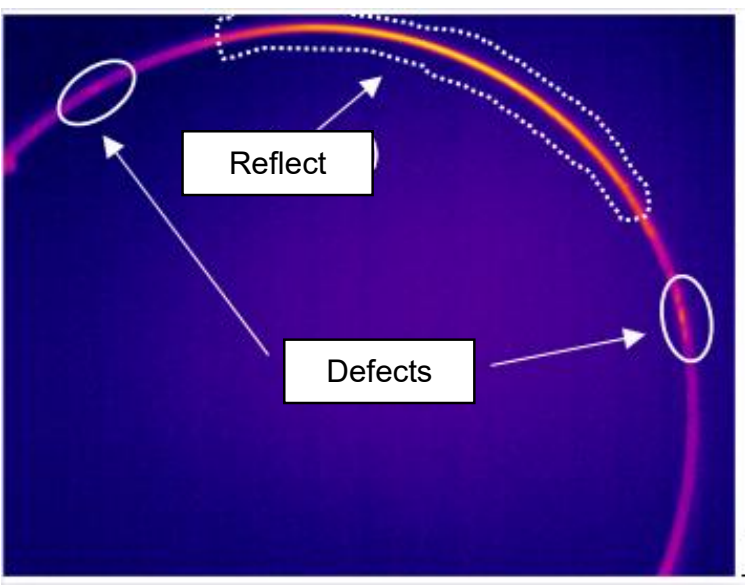

Fig. 11. Used arches

Direct observation makes it difficult to detect defects on the arcs for two reasons: the weakness of the thermal contrast and the presence of reflections, which makes identification difficult when defects exist. Thus in Figure 11 (used), it is noticed that at least 2 thermally active areas of comparable intensities. However, a more careful observation shows us that we are dealing in one case with a reflection identified by a soft outline, and in other cases with surface defects identified by sharp and irregular contours. Static analysis on the series of samples studied was made difficult and inconclusive. The cause of this phenomenon can be explained in a simple way using the equation of thermography :

$$
L(\lambda, \perp)=\tau_{a t m, \lambda, \perp} \cdot \epsilon_{\lambda, \perp} \cdot \sigma \cdot T_{e c h}^{4}+\rho_{e c h, \lambda, \perp} \cdot \epsilon_{a m b, \lambda, \perp} \cdot \sigma \cdot T_{a m b}^{4}+\left(1-\tau_{a t m, \lambda, \perp}\right) \cdot \sigma \cdot T_{a i r}^{4}
$$

With respectively $T_{-}(\lambda, \perp), \varepsilon_{-}(\lambda, \perp), \rho_{-}(\lambda, \perp)$ the transmissivity, emissivity and directional spectral reflectiveness of the different media (atmosphere, samples, ambient). T medium temperatures and $\sigma$ Stefan-Boltzmann's constant.

It is known that the signal measured by the camera can have several causes :

\begin{tabular}{|l|c|c|}
\hline - The sample's own emission (thermal effect) & Sample contribution & $\tau_{a t m} \epsilon \sigma T_{e c h}^{4}$ \\
\hline $\begin{array}{l}\text { - Multiple reflections of the ambient environment on the } \\
\text { sample (Radiative effect) }\end{array}$ & $\begin{array}{c}\text { Contribution of the } \\
\text { environment }\end{array}$ & $\rho_{e c h} \epsilon_{a m b} \sigma T_{a m b}^{4}$ \\
\cline { 1 - 1 } - Atmospheric emission & $\left(1-\tau_{a t m}\right) \sigma T_{a i r}^{4}$ \\
\hline
\end{tabular}

However, in the static regime, the orders of magnitude between the actual emission of the sample and the environmental contributions are comparable, which has the effect of exaggerating the relative importance of the radiative 
and atmospheric effects. On the other hand, in a dynamic regime (joule heating here) the own emission of the sample is preponderant compared to the other contributions because it follows a rapid evolution in T4 which has the effect of reducing their parasitic contribution in the 'analysis.

\subsubsection{Dynamic analysis}

In the table below, it would be observed that the thermal images of a new and used sample coming from the same manufacturer subjected to a thermal disturbance (joule effect). It may be concluded that the new samples have a thermally homogeneous surface in contrast to the used sample.

Table 3. Comparaison arcs American ortho en dynamique

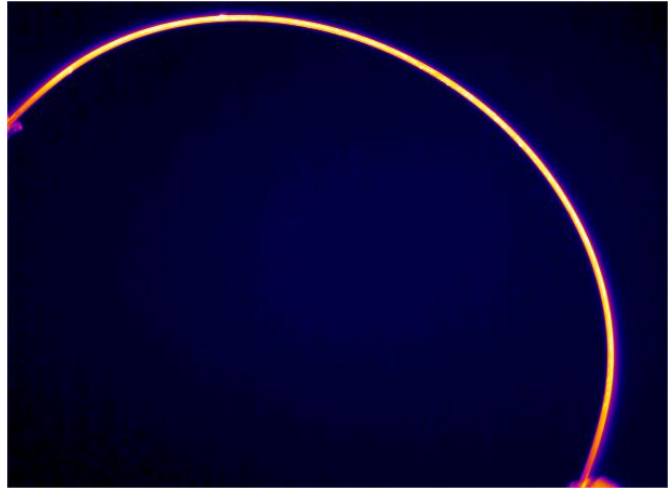

Fig. 12. New arch

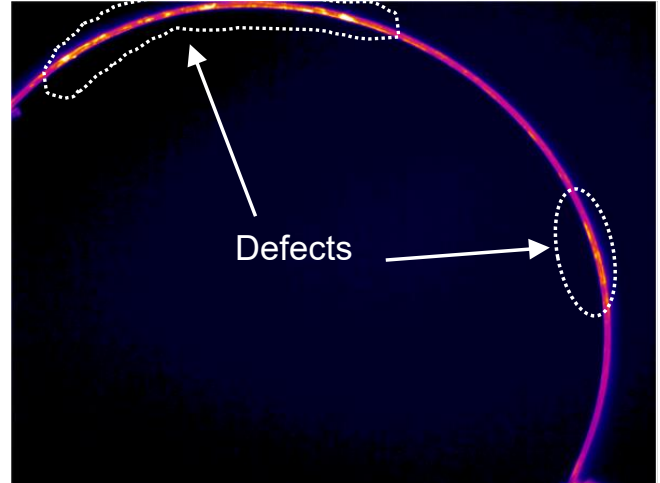

Fig.13. Used arch

The following table shows two new and used samples from another manufacturer (Azdent) which subjected to the same thermal disturbance and was observed in the presence of temperature inhomogeneity on the two arcs. It would be noted that the used sample has more thermally active zones.

Table 4. Comparaison arcs Azdent en dynamique

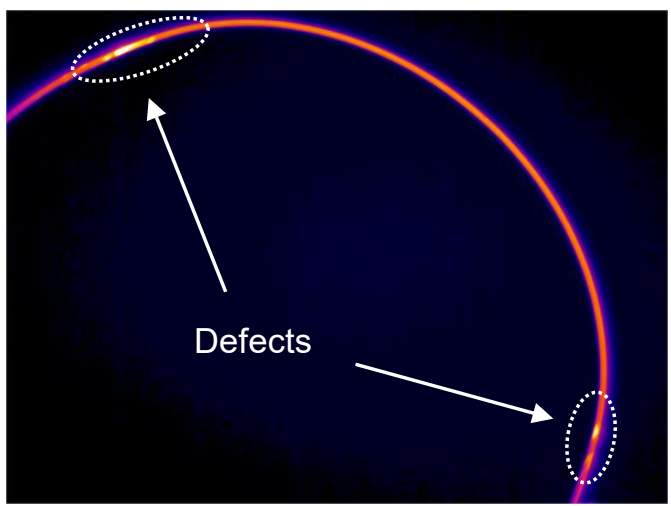

Fig. 14. New arch

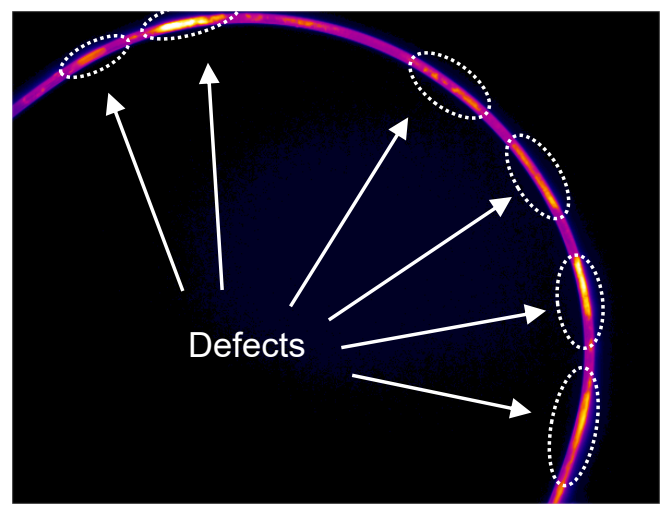

Fig. 15. Used arch

These observations on a number of samples indicate that as a rule the new samples may have defects but in a limited number in comparison to the used samples having more defects. Note also that the dynamic regime can identify a larger number of defects. 


\subsection{Quantitative approach}

In this analysis, we are interested in the number of defects and their characterization. We will study the thermograms obtained in a dynamic mode.

\subsubsection{Dynamic regime analysis}

The fault count was performed as previously indicated using the ImageJ software. As can be seen in the following table the number of defects has been accounted for, as well as the area of defects that has been estimated.

Table 5. Defect characterization

\begin{tabular}{|l|l|l|l|l|}
\hline Status & \multicolumn{2}{|c|}{ New } & \multicolumn{2}{c|}{ Used } \\
\hline Type & $\begin{array}{l}\text { Number of } \\
\text { defects }\end{array}$ & Total area & $\begin{array}{l}\text { Number of } \\
\text { defects }\end{array}$ & Total area \\
\hline 1 & 3 & 84 & 15 & 86 \\
\hline 2 & 0 & 0 & 24 & 166 \\
\hline 3 & 4 & 4 & 10 & 18 \\
\hline 4 & 9 & 13 & 6 & 16 \\
\hline 5 & 5 & 6 & 19 & 167 \\
\hline 6 & 5 & 5 & 12 & 41 \\
\hline 7 & 5 & 14 & 12 & 411 \\
\hline 8 & 5 & 98 & 23 & 225 \\
\hline 9 & 2 & 80 & 18 & 385 \\
\hline 10 & 3 & 3 & 13 & 38 \\
\hline 11 & 6 & 357 & 9 & 226 \\
\hline 12 & 2 & 167 & 17 & 226 \\
\hline 13 & 5 & 45 & 26 & 194 \\
\hline 14 & 0 & 0 & 15 & 104 \\
\hline 15 & 3 & 19 & 10 & 205 \\
\hline 16 & 2 & 77 & 27 & 188 \\
\hline 17 & 4 & 179 & 24 & 254 \\
\hline 18 & 8 & 30 & 15 & 153 \\
\hline
\end{tabular}

First, we wanted to verify the previous observation that used samples had a higher number of defects. The histogram below clearly confirms this observation. For each variant of the same type of sample (same manufacturer, same diameter) the ratio number of used defect / number of new defect can be greater than 4 in certain cases (see for example type 8 and 14).

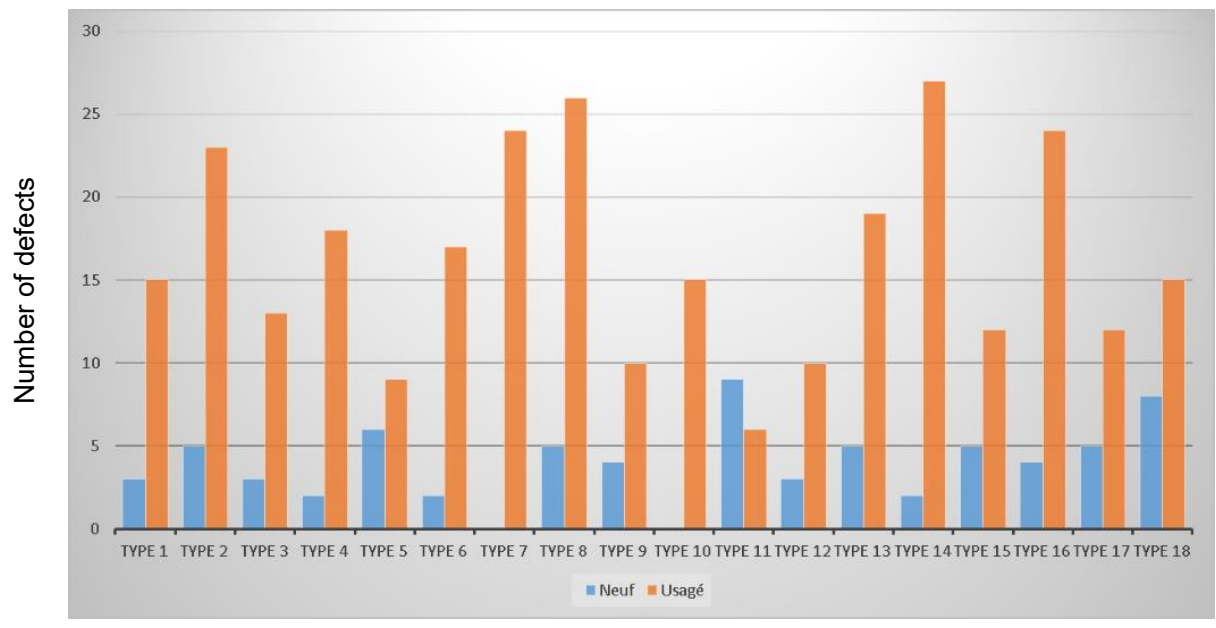

Fig. 16. Comparison of the number of new/used faults

Then studied the distribution of the samples according to a multicriterial approach, we crossed the two measured parameters (number of defects and area of the defects) and observed the distribution of the points of measurement. Once 
again we noticed a clear distribution when these two criteria are crossed: we have two distinct distributions of the measurement points (see graph below) :

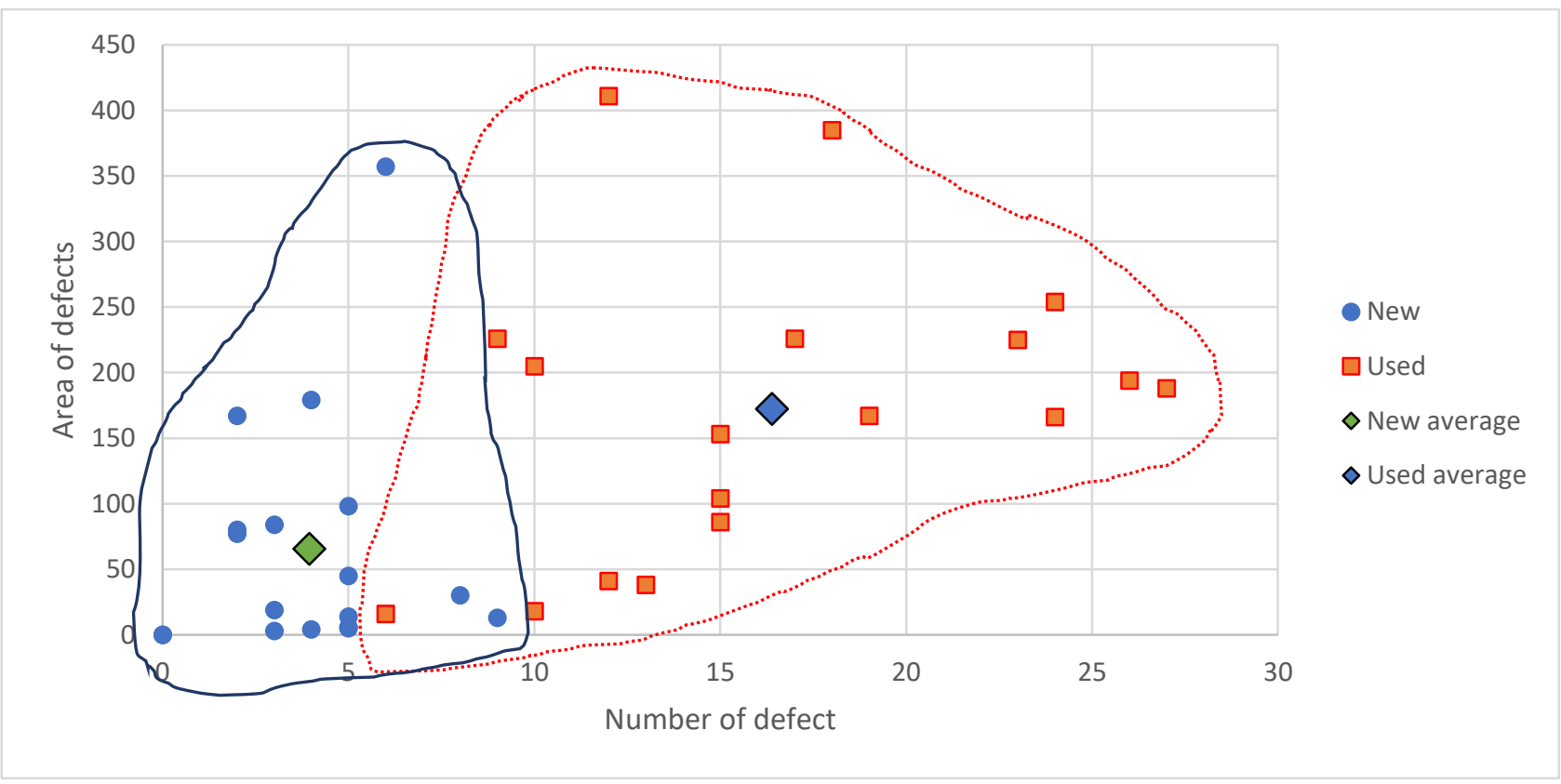

Fig. 17. Multi-criteria analysis

For the new samples, we observed a grouping in the lower left part of the graph. However, this was not the case in the used samples which were grouped in the right part in a slightly higher position, which means that the new samples have on average a number of defects lower than those present in the used samples. Moreover, the areas of these defects, as present in the new samples, are on average slightly less than those found of the used arcs. In summary, we have more defects, more in the used which is consistent with the intuition that we can have and the observations made in static mode.

The table below shows that the differentiation criterion between the two types of arcs is the number of defects more than their size. As seen in the following table when comparing the difference between the barycenter of the two series and the standard deviations of the total population of the samples, it was found that this ratio is greater along the axis corresponding to the parameter "number of defects".

Table 6. Recap

\begin{tabular}{|l|l|l|l|l|l|}
\hline & New samples & $\begin{array}{l}\text { Used } \\
\text { samples }\end{array}$ & $\begin{array}{l}\text { Statistics on } \\
\text { all samples }\end{array}$ & $\begin{array}{l}\text { Barycentric } \\
\text { deviation }\end{array}$ & $\begin{array}{l}\text { Ratio of barycentric } \\
\text { deviation to standard } \\
\text { deviation of total } \\
\text { population (\%) }\end{array}$ \\
\hline $\begin{array}{l}\text { Average number of } \\
\text { defects }\end{array}$ & 3,9 & 16,4 & 10,2 & 12,4 & 162 \\
\hline $\begin{array}{l}\text { Standard deviation } \\
\text { number of defects }\end{array}$ & 2,4 & 6,3 & 7,7 & & \\
\hline $\begin{array}{l}\text { Average defect area } \\
\left(\mathrm{px}^{2}\right)\end{array}$ & 65,6 & 172,4 & 119 & 106,8 & 94 \\
\hline $\begin{array}{l}\text { Defect area standard } \\
\text { deviation }\left(\mathrm{px}^{2}\right)\end{array}$ & 91,6 & 112,5 & 113 & & \\
\hline
\end{tabular}




\section{Conclusion}

This study made it possible to differentiate between two sets of samples of orthodontic arches, new and used, and to quantify their state of deterioration by studying their thermal responses in static and dynamic regimes (with joule heating).

Static analysis did not significantly and reliably differentiate the two sample series due to the preponderance of detection artifacts related to ambient conditions such as reflection. The presence of many reflections did not allow to effectively differentiate the two batches of samples. To correct this, it was necessary to warm the samples to reduce the influence of these effects. For this, the samples were subjected to an electric current of a power of $4 \mathrm{~W}$ for $15 \mathrm{~s}$. Thermal disruption allowed the Joule samples to heat up and thus increase the thermal contrast of the defects while reducing the influence of the reflections.

The dynamic analysis showed that the new samples had fewer defects than the used ones as may be expected. An additional quantitative analysis using the ImageJ software made it possible to count these defects and measure their surface area. The crossing of these parameters showed that the two series of samples could be correctly differentiated by these two quantities: the used samples have a significantly greater number of defects, and their average size seems slightly larger than that of the new samples.

In conclusion, we can say that stimulated infrared thermography is an effective method of analysis that allowed us to highlight the presence of alteration in the orthodontic arches. This work is not complete yet and work is in progress to identify the origin of these alterations. 